\title{
Eficacia y seguridad de Tadalafilo en dos regímenes de dosificación diferentes en pacientes españoles con disfunción eréctil: Resultados procedentes del estudio SURE realizado en 14 países europeos
}

\author{
Martín-Morales $\mathrm{A}^{1}$, Moncada Iribarren $\mathrm{I}^{2}$, Cruz Navarro $\mathrm{N}^{3}$, Sanz Terrada $\mathrm{B}^{4}$, \\ Cassinello Hervás $\mathrm{A}^{4}$, Chan $\mathrm{M}^{5}$, Casariego García-Lubén $\mathrm{J}^{4}$. \\ ${ }^{1}$ Servicio de Urología. H. Carlos Haya. Málaga. ${ }^{2}$ Servicio de Urología. H. Gregorio Marañón. Madrid. \\ ${ }^{3}$ Servicio de Urología. Hospital Virgen del Rocío. Sevilla. ${ }^{4}$ Lilly S.A. Alcobendas, Madrid. \\ ${ }^{5}$ Eli Lilly Canada Inc, Toronto, Ontario, Canadá. \\ Actas Urol Esp. 2006;30(8):791-800
}

\begin{abstract}
RESUMEN
EFICACIA Y SEGURIDAD DE TADALAFILO EN DOS REGIMENES DE DOSIFICACIÓN DIFERENTES EN PACIENTES ESPAÑOLES CON DISFUNCIÓN ERÉCTIL: RESULTADOS PROCEDENTES DEL ESTUDIO SURE REALIZADO EN 14 PAÍSES EUROPEOS

Fundamento y objetivo: Comparar eficacia y seguridad de tadalafilo 20 mg administrado 3 veces/semana ("pautado") vs. "a demanda" en una cohorte de españoles con disfunción eréctil (DE) aprovechando que su efecto permanece hasta 36 horas postdosis.

Material y método: Los 418 españoles participantes en el estudio europeo SURE (multicéntrico, cruzado y abierto en 4.262 pacientes), se distribuyeron aleatoriamente a una de las siguientes secuencias: tadalafilo $20 \mathrm{mg}$ "a demanda" (D) durante 5-6 semanas seguido de tadalafilo $20 \mathrm{mg}$ pautado, 3 veces por semana (P) otras 5-6 semanas; o la inversa. Al finalizar los pacientes eligieron el régimen que preferían en una extensión del estudio.

Resultados: Tadalafilo en ambos regímenes mejoró la función eréctil (FE) respecto a la basal. El régimen $\mathrm{P}$ obtuvo significativamente mejores puntuaciones que el D en ciertos dominios del cuestionario IIEF (p.ej. deseo sexual), y más pacientes (69,3\% vs $64,3 \%, P<0,05$ ) normalizaron su FE (puntuación del dominio de FE del IIEF $\geq 26$ )), y alcanzaron mayor porcentaje de intentos de coito exitosos (SEP 3) $(75,7 \%$ vs $72,2 \% ;(P<0,05)$ con el P. No obstante, más pacientes prefirieron recibir tadalafilo en régimen D que $\mathrm{P},(55,9 \%$ vs. $44,1 \%, P<0,05)$. Tadalafilo en ambos regimenes fue bien tolerado. Los acontecimientos adversos más frecuentes $(\geq 5 \%)$ relacionados con el tratamiento fueron cefalea, dispepsia y dolor de espalda. No hubo diferencias significativas en la incidencia de los mismos.

Conclusiones: Tadalafilo $20 \mathrm{mg}$ es eficaz en el tratamiento de la DE en cualquiera de los regimenes de administración ("a demanda" ó 3 veces/semana) empleado, siendo bien tolerado. Los pacientes pueden escoger la pauta de administración mas adecuada.
\end{abstract}

Palabras clave: Tadalafilo/administración y dosis/uso terapéutico. Impotencia/tratamiento farmacológico. Satisfacción del paciente. Inhibidores de la fosfodiesterasa. Estudio comparativo. Estudios cruzados.

\section{ABSTRACT}

EFFICACY AND SAFETY OF TWO DOSING REGIMENS WITH TADALAFIL IN SPANISH MEN WITH ERECTILE DYSFUNCTION: RESULTS FROM THE SURE STUDY IN 14 EUROPEAN COUNTRIES

Objetive: To compare the efficacy and safety of tadalafil $20 \mathrm{mg}$ administered 3 times/week (SCH) vs. on demand (OD) in a cohort of Spanish men with erectile dysfunction (ED), since Tadalafil period of responsiveness lasts up to 36 hours post-dosing.

Material and methods: The 418 Spanish patients participating in the European multicenter, crossover, open-label SURE clinical trial (comprising $4262 \mathrm{men}$ ) were randomly assigned to one of the treatment sequences: tadalafil $20 \mathrm{mg}$ SCH for 5-6 weeks followed by tadalafil $20 \mathrm{mg}$ OD for 5-6 weeks, or the inverse sequence. At completion, patients were asked to select the regimen they preferred to receive in an extension phase.

Results: In both regimens, tadalafil led to a similar improvement in erectile function compared to baseline. However, the SCH regimen showed statistically significant higher scores for several IIEF questions (i.e. sexual desire domain). Normal erectile function (IIEF EF domain score 226 ) was achieved by $69.3 \%$ of patients on SCH and $64.3 \%$ on OD, with a sexual intercourse success rate (SEP3) of $75.6 \%$ and $72.2 \%$ respectively $(\mathrm{p}<0.05)$. Nevertheless, more patients preferred to receive tadalafil OD for the extension phase $(55.9 \%$ vs $44.1 \%$, p $<0.05)$. Tadalafil was well tolerated in both regimens. The most common TEAEs $(\geq 5 \%)$ were headache, dyspepsia and back pain. There were no clinically significant differences in the incidence of TEAEs between regimens. Conclusions: Tadalafil $20 \mathrm{mg}$ is efficacious and well tolerated for the treatment of ED, regardless the regimen of administration (OD or $\mathrm{SCH})$. Patients can choose the pattern of administration that fits better with their expectations.

Keywords: Tadalafil/administration \& dosage/ therapeutic use. Impotence/drug therapy. Patient satisfaction. Phosphodiesterase inhibitors. Drug Administration Schedule. Comparative Study. Cross-Over Studies. 
$\mathrm{L}^{\mathrm{a}}$ a disfunción eréctil (DE), incapacidad para conseguir o mantener una erección con suficiente rigidez para permitir una relación sexual satisfactoria, afecta en España a un 12,1\% de la población masculina en mayor o menor grado ${ }^{1}$.

La mayoría de los pacientes con disfunción eréctil presentan un origen inicialmente orgánico, al que posteriormente se suman un componente psicoafectivo y un sentimiento de miedo al fracaso que potencian y agravan la disfunción ${ }^{2}$. Los inhibidores de la fosfodiesterasa 5 (PDE5) son los tratamientos considerados de primera elección para la DE en la mayoría de los pacientes debido a su eficacia y seguridad ${ }^{3}$.

Estos fármacos permiten restaurar la capacidad eréctil inhibiendo casi por completo la actividad de la PDE5, pero presentan entre si diferencias bioquímicas que se traducen en diferencias en cuanto a la rapidez de inhibición, la duración de acción, las interacciones con otros fármacos o los efectos adversos.

Por tanto, el abordaje médico de la DE en el momento actual ha de considerar las preferencias y necesidades de los pacientes ${ }^{4}$ y ofrecer la alternativa existente que mas se adecue a cada paciente ${ }^{5-6}$.

Tadalafilo se encuentra comercializado en más de 100 países en todo el mundo, que incluyen los Estados Unidos y Europa. La mayoría de los estudios clinicos realizados hasta la fecha con tadalafilo han evaluado su uso administrado "a demanda”, es decir a discreción del paciente previamente a la relación sexual esperada. Un análisis integrado de los datos de 11 estudios con tadalafilo ${ }^{7}$ observó que el 54\% de los pacientes con DE que tomaron tadalafilo $20 \mathrm{mg}$ "a demanda" durante un periodo de 12 semanas recobraron la normalidad de la función eréctil basada en las puntuaciones obtenidas $(\geq 26)$ en el Índice Internacional de Función Eréctil (IIEF). Adicionalmente, estudios recientes indican que tadalafilo permite establecer una ventana de respuesta eréctil que abarca desde los 16 minutos $^{8}$ hasta las 36 horas de duración del efecto tras su administración ${ }^{7-14}$. Por ello, tadalafilo podría aportar una cobertura casi continua al tomarlo de forma regular, permitiendo a los pacientes y sus parejas elegir el mejor momento en el que desean tener la relación sexual, despreocupándose del tiempo disponible para mantener relaciones sexuales.
Este estudio recoge los resultados observados en una cohorte de pacientes españoles con DE que participaron en el estudio SURE (Scheduled Use versus on demand Regimen Evaluation) realizado en 14 países europeos: El estudio SURE tenía como objetivo valorar la preferencia de los pacientes por una pauta de administración alternativa, de 3 veces por semana, frente a la hasta ahora tradicional forma de consumo "a demanda" de este tipo de fármacos. Los resultados globales de este estudio ya han sido comunicados por Mirone et $\mathrm{al}^{14}$.

\section{MATERIAL Y MÉTODOS}

Pacientes y diseño del estudio

Se invitó a participar en el estudio SURE a 418 pacientes con disfunción eréctil reclutados en 33 centros españoles. Se trató de un estudio multicéntrico, abierto y de diseño cruzado de 12 semanas de duración realizado en 14 países europeos (4.262 pacientes en total) para determinar la preferencia de pacientes con disfunción eréctil por uno de los siguientes regímenes de tratamiento con tadalafilo $20 \mathrm{mg}$ "a demanda" o "pautado" 3 veces/semana ${ }^{15}$. "A demanda" consistía en la administración a discreción del paciente de tadalafilo previamente a la relación sexual esperada pero sin exceder el límite máximo de una dosis al día. "Pautado" consistió en la administración regular de tadalafilo 3 veces por semana (lunes, miércoles y viernes / martes, jueves y sábado) independientemente de la actividad sexual espontánea.

Los participantes reclutados eran varones $>18$ años de edad con historia de disfunción eréctil de al menos de 3 meses de duración, que para ser incluidos deberían mantener una relación estable con la misma pareja femenina durante todo el estudio. Pudieron participar en este estudio un amplio abanico de pacientes con disfunción eréctil de diversa afectación funcional (leve a severa) y etiología (psicógena, orgánica y mixta). En el periodo inicial de selección los pacientes deberían realizar al menos 4 tentativas de coito con su pareja. Además se comprometían a no utilizar ningún tratamiento para la $\mathrm{DE}$ durante las fases de pretratamiento y tratamiento, así como durante las 96 horas posteriores a la última visita del estudio. 
No pudieron participar en el estudio aquellos pacientes que recibieran tratamiento con nitratos, quimioterápicos, terapia antiandrogénica, o tuvieran historial de insuficiencia cardiaca congestiva sintomática.

Se permitió la participación en el estudio a varones que hubiesen estado con anterioridad en tratamiento con algún inhibidor de fosfodiesterasa 5 .

Tras un periodo de pretratamiento de 3-4 semanas en el que se seleccionó a los pacientes, éstos se distribuyeron de forma aleatoria para recibir tadalafilo $20 \mathrm{mg}$ en régimen "a demanda" antes de la actividad sexual hasta un máximo de una dosis diaria o pautado (3 veces/semana) durante 5-6 semanas. Tras una semana de lavado, se cruzaron para recibir el régimen alternativo durante otras 5-6 semanas. El régimen de 3 veces por semana se subdividió a su vez en dos subgrupos: subgrupo A (Lunes, Miércoles y Viernes) y subgrupo B (Martes, Jueves y Sábados). Al finalizar, se dio a los pacientes la opción de elegir el tratamiento preferido (a demanda ó 3 veces/ semana), para continuar como mínimo durante otras 2 semanas en una extensión del estudio (Fig. 1).

\section{Variables de valoración del estudio}

Preferencia: La variable principal del estudio fue la valoración de la preferencia del paciente por uno $\mathrm{u}$ otro régimen en función de la respuesta de los pacientes al final del estudio cruzado a la "Pregunta de Preferencia del Tratamiento (PPT)“:¿que régimen de tratamiento prefiere?: ("A demanda" ó 3 veces/semana) que se contestó al finalizar el segundo periodo de tratamiento cruzado de 5-6 semanas.

Eficacia: Como variables secundarias se evaluaron la eficacia y la satisfacción del paciente con tadalafilo y su relación con el régimen preferido mediante los cuestionarios IIEF y SEP.

Para evaluar los efectos de tadalafilo sobre la función eréctil se emplearon el Índice Internacional de la Función Eréctil (IIEF) y el diario del paciente Perfil del Encuentro Sexual (SEP). El IIEF es un cuestionario validado multidimensional, de autoevaluación que consta de 15 preguntas que evalúan 5 dominios de la función sexual masculina: la función eréctil, la función orgásmica, el deseo sexual, la satisfacción durante el coito, y la satisfacción global durante las 4 ultimas semanas ${ }^{15}$. El SEP es un diario que consta de 5 preguntas en donde se valoran los resulta-

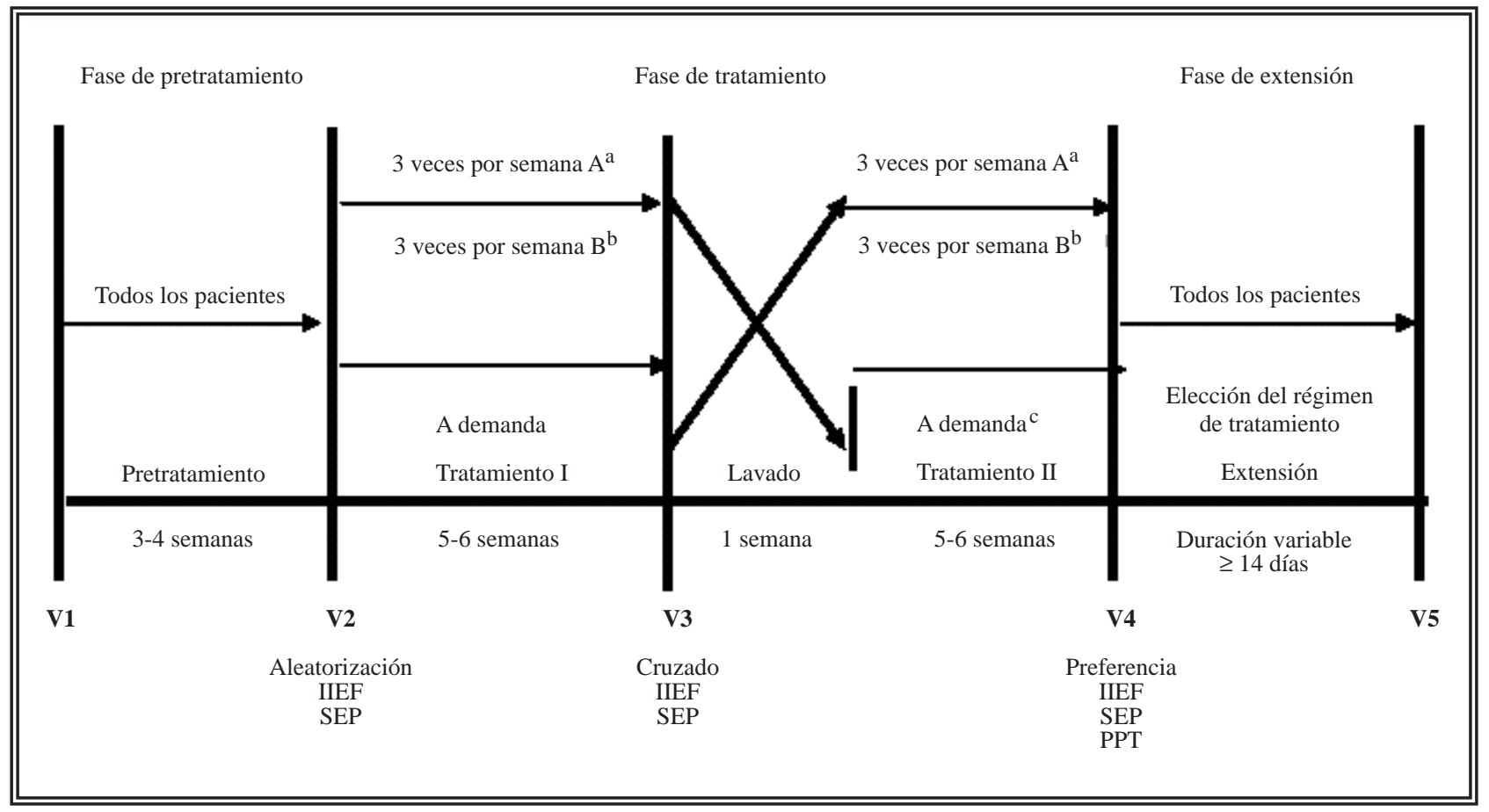

FIGURA 1. Diseño del estudio SURE. 
dos de cada intento de coito y que el paciente cumplimenta tras cada tentativa de relación sexual.

La eficacia sobre las erecciones se evaluó según el cambio experimentado desde el inicio al final del studio en el dominio de FE del IIEF y el porcentaje de respuestas afirmativas a las preguntas $2^{a}$ (capacidad de introducir el pene en la vagina) y $3^{\text {a }}$ (capacidad de mantener las erecciones el tiempo suficiente para mantener una relación sexual con éxito) del cuestionario SEP.

Por otra parte, la satisfacción del paciente con las relaciones sexuales se midió a través del cambio en las puntuaciones obtenidas en el dominio de satisfacción del IIEF desde el inicio al final del estudio, así como a través del porcentaje de respuestas "si" a las preguntas $4^{\text {a }}$ (satisfacción con la dureza de la erección) y $5^{\text {a }}$ (satisfacción global con la experiencia sexual) del diario SEP.

Seguridad: En el análisis de seguridad se incluyó a todos los pacientes incluidos en la asignación aleatoria. Los acontecimientos adversos aparecidos durante el tratamiento (AAs), definidos como aquellos que se daban por primera vez o que se agravaban después de la evaluación basal, se catalogaron con los términos del Medical Dictionary for Regulatory Activities (MedDRA versión 5.0).

Análisis estadístico: Los análisis del estudio se realizaron según el criterio de intención de tratar. La variable de valoración principal fue la PPT y el análisis incluyó a todos los pacientes que completaron el cuestionario. La hipótesis nula fue que la proporción de pacientes que preferían la pauta de administración a demanda y 3 veces por semana de tadalafilo era la misma. Se utilizó la prueba de $z$ de una muestra, con una significación bilateral de 0,05 para analizar la hipótesis nula. Para todas las demás variables de eficacia, las puntuaciones de dominios del IIEF y las preguntas 1-5 del SEP, los análisis incluyeron a todos los pacientes para los que se disponía de una observación basal y de al menos una observación posterior a la basal. $\mathrm{El}$ cambio respecto al valor basal en las variables de eficacia se analizó mediante un modelo de ANOVA para datos cruzados. Ese modelo incluía términos fijos para la pauta de tratamiento (a demanda ó 3 veces por semana), el valor basal de la variable de eficacia, centro, periodo, y situación basal con el tratamiento, y un término aleatorio para el paciente. En cualquiera de los modelos, si la interacción no era significativa $(p>0,10)$, se retiraba del modelo. No se incluyó efecto de arrastre puesto que el lavado farmacológico de una semana entre los periodos de tratamiento se consideraba suficiente para eliminar todo efecto farmacodinámico de tadalafilo del periodo anterior. En la fase de tratamiento de este estudio, se valoró la comorbilidad registrada en la primera visita y los posibles acontecimientos adversos registrados antes de la asignación aleatoria para establecer los AAs basales en ambos periodos de tratamiento. Se utilizaron todos los AAs persistentes al final del segundo periodo de tratamiento para establecer los AAs basales para la fase de extensión.

\section{RESULTADOS}

\section{Características de los pacientes}

418 pacientes españoles reclutados fueron incluidos en la asignación aleatoria para recibir una u otra secuencia de tratamiento cruzado (Fig. 2). De éstos pacientes, 366 completaron el estudio $(87,6 \%)$. De los 52 pacientes $(12,4 \%)$ que abandonaron el estudio un 5\% lo hizo a causa de los acontecimientos adversos, un $3,1 \%$ por conflictos personales o decisión del paciente, un $2,4 \%$ por falta de eficacia, un $1,5 \%$ por no cumplir los criterios de inclusión del protocolo o por violación de éste y el $0,5 \%$ restante por pérdida de seguimiento. Las características demográficas de los pacientes en las dos secuencias de tratamiento quedan recogidas en la Tabla 1.

La edad media de los pacientes fue de 54,9 años. La mayoría $(89,5 \%)$ presentaban historial de más de un año de duración de disfunción eréctil, cuya etiología en un alto porcentaje era de origen orgánico $(47,4 \%)$ o mixto $(38 \%)$. La severidad de la DE al comienzo del estudio fue leve en el $42,6 \%$ de los pacientes, moderada en el $25,6 \%$ y severa en el $31,8 \%$.

\section{Variable principal: preferencia del paciente (PPT)}

Un total de 374 pacientes respondieron al final de la fase de tratamiento a la pregunta: "¿Qué régimen de tratamiento prefiere?: ("A de- 


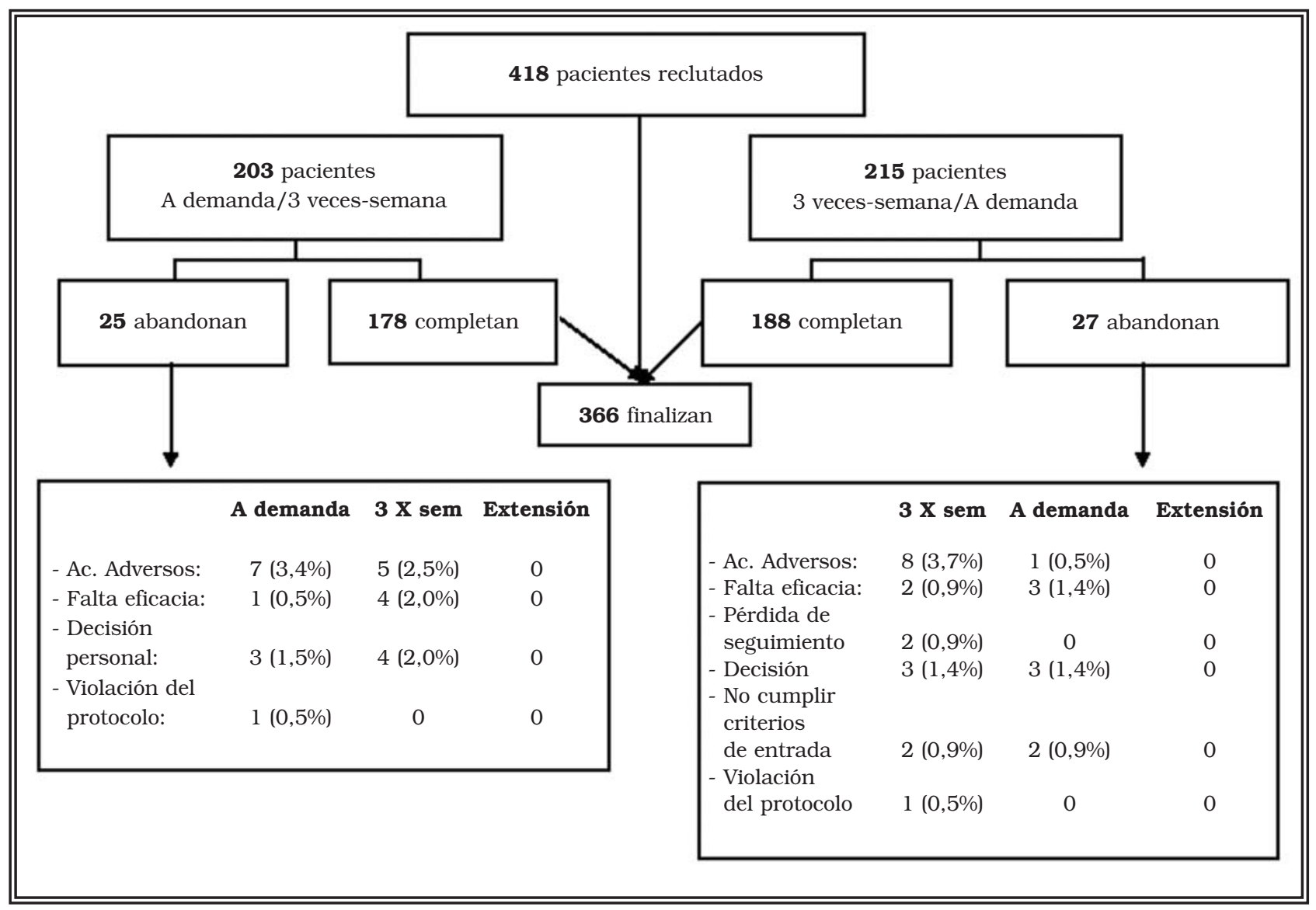

FIGURA 2. Disposición de los pacientes.

Tabla 1

Características demográficas de los pacientes y causas de la disfunción eréctil (DE)

\begin{tabular}{lr}
\hline & $\begin{array}{c}\text { Total } \\
\text { (n = 418) }\end{array}$ \\
\hline $\begin{array}{l}\text { Edad media, años (Rango) } \\
\text { Raza, n (\%) }\end{array}$ & $54,9(25-77)$ \\
$\quad$ Caucásica & $418(100)$ \\
Etiología DE, n (\%) & \\
$\quad$ Psicógena (\%) & $61(14,6)$ \\
$\quad$ Orgánica (\%) & $198(47,4)$ \\
$\quad$ Mixta (\%) & $159(38,0)$ \\
Severidad DE, n (\%) & \\
$\quad$ Leve (\%) & $178(42,6)$ \\
$\quad$ Moderada (\%) & $107(25,6)$ \\
$\quad$ Severa (\%) & $133(31,8)$ \\
Duración DE, n (\%) & \\
$\quad \geq 3$ Meses \& $<1$ año & \\
$\quad \geq 1$ año & $44(10,5)$ \\
Puntuación media dominio FE del IIEF & \\
(DE) &
\end{tabular}

manda"/3 veces/semana)". En base al porcentaje de respuestas, un número significativamente mayor de pacientes prefirieron recibir tadalafilo en régimen "a demanda" $(55,9 \% ; n=209)$ que en régimen 3 veces/semana $(44,1 \% ; n=165)$; $P<0,05)$ en la fase de extensión del estudio.

\section{Variables secundarias: IIEF y SEP}

Ambos regímenes de administración fueron eficaces. En ambos regímenes, el tratamiento con tadalafilo condujo a una mejoría similar del dominio de función eréctil del IIEF en comparación con el periodo basal, de manera que los que recibieron el régimen a demanda mejoraron sus puntuaciones medias basales de 15,1 a 24,7 al final del tratamiento y los que recibieron el régimen 3 veces/semana de 15,0 basal a 25,1 tras la fase de tratamiento $(P>0,05)$. Dicha mejoría fue más notable en aquellos pacientes que padecian una disfunción eréctil severa (puntuación en el Dominio FE del IIEF $\leq 10$ ) al iniciar el estudio, tal 
como se observa en el cambio medio en las puntuaciones del dominio de función eréctil reflejadas en la Figura 3.

Concretamente, un porcentaje significativamente mayor de pacientes normalizaron su función eréctil (puntuación del dominio de función eréctil del IIEF $\geq 26$ ) al recibir tadalafilo en régimen de 3 veces/semana que al recibirlo como tratamiento "a demanda" $(69,3 \%$ vs. $64,3 \%$, respectivamente) (Fig. 4), incluidos aquellos en los que la disfunción era más grave (puntuación en

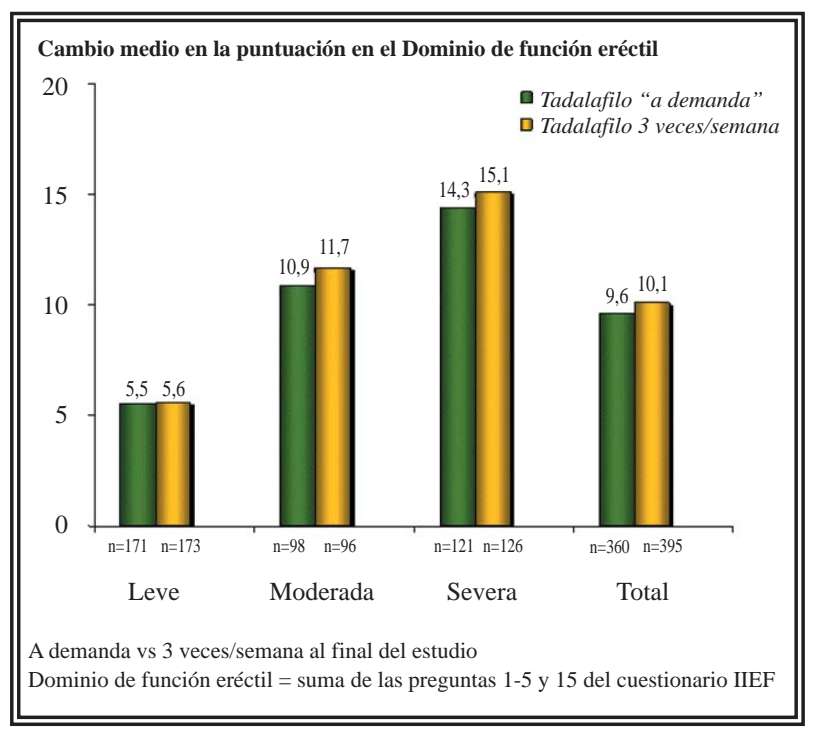

FIGURA 3. Cambio medio respecto al valor basal en el dominio de función eréctil del IIEF estratificado en función de la severidad de la disfunción eréctil a nivel basal.

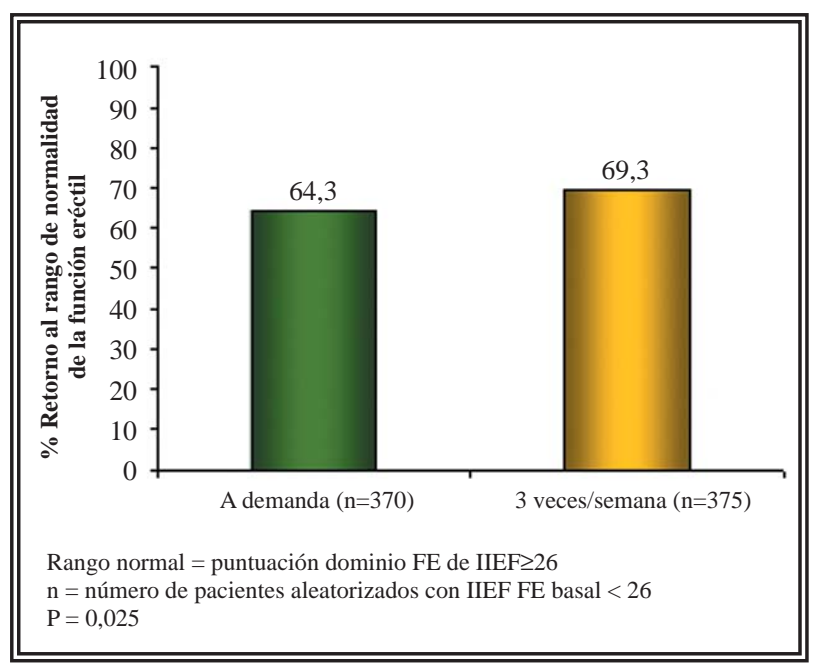

FIGURA 4. Porcentaje de pacientes que alcanzan al final del tratamiento una puntuación en el dominio FE del IIEF comprendido dentro del rango normal de función eréctil. el dominio $\mathrm{FE}$ del $\mathrm{IIEF}<10$ ) en el periodo basal ( $57,9 \%$ con tadalafilo 3 veces/semana vs. $49,6 \%$ con tadalafilo a demanda) $(P<0,05)$. Asimismo, en el dominio de deseo sexual el tratamiento con tadalafilo 3 veces/semana condujo a una mejoría en la puntuación media basal de 6,4 a 7,3 que fue significativamente superior $(P<0,05)$ a la observada con tadalafilo "a demanda" (de 6,4 a 7,1) (Fig. 5).

También, al final del tratamiento el porcentaje de intentos de coito exitosos (SEP3) fue del $75,6 \%$ y del $72,2 \%$, con los regímenes 3 veces/semana y "a demanda" respectivamente $(P<0,05)$ (Figura 5). Y de la misma manera en el resto de las preguntas individuales del cuestionario SEP las diferencias fueron significativas a favor del régimen programado (Fig. 6).

Los pacientes mostraron mayor satisfacción con la pauta de dosificación de tres veces/semana según las respuestas a las preguntas $4^{\mathrm{a}}$ (¿Está satisfecho con la dureza de sus erecciones?) y $5^{\text {a }}$ (¿Está satisfecho en general con sus relaciones sexuales?) del cuestionario del diario SEP $(P=0,001)$. El cambio medio desde el inicio al final del estudio en el dominio de satisfacción general del cuestionario IIEF fue de 3,0 y 2,9 para los regimenes 3 veces/semana y "a demanda" respectivamente ( $\mathrm{P}>0,05)$ (Fig. 5).

\section{Seguridad}

Tadalafilo fue bien tolerado en ambos regímenes de administración. Los acontecimientos adversos más frecuentes (incidencia $\geq 5 \%$ ) fueron dispepsia, cefalea y dolor del espalda. En la Tabla 2 se muestran aquellos con una incidencia $\geq 2 \%$.

Los pacientes en el regimen de 3 veces/ semana tomaron un mayor número de dosis por semana que durante el tratamiento "a demanda" (media 2,3 vs. 1,7) como se muestra en la Tabla 3. No se observaron diferencias clínicamente significativas en la incidencia de AAs entre ambos regímenes. Tampoco se observó ninguna alteración en la frecuencia cardiaca ni en la PA, en ninguno de los grupos de tratamiento. En un 5\% de los pacientes los acontecimientos adversos condujeron al abandono del estudio. 


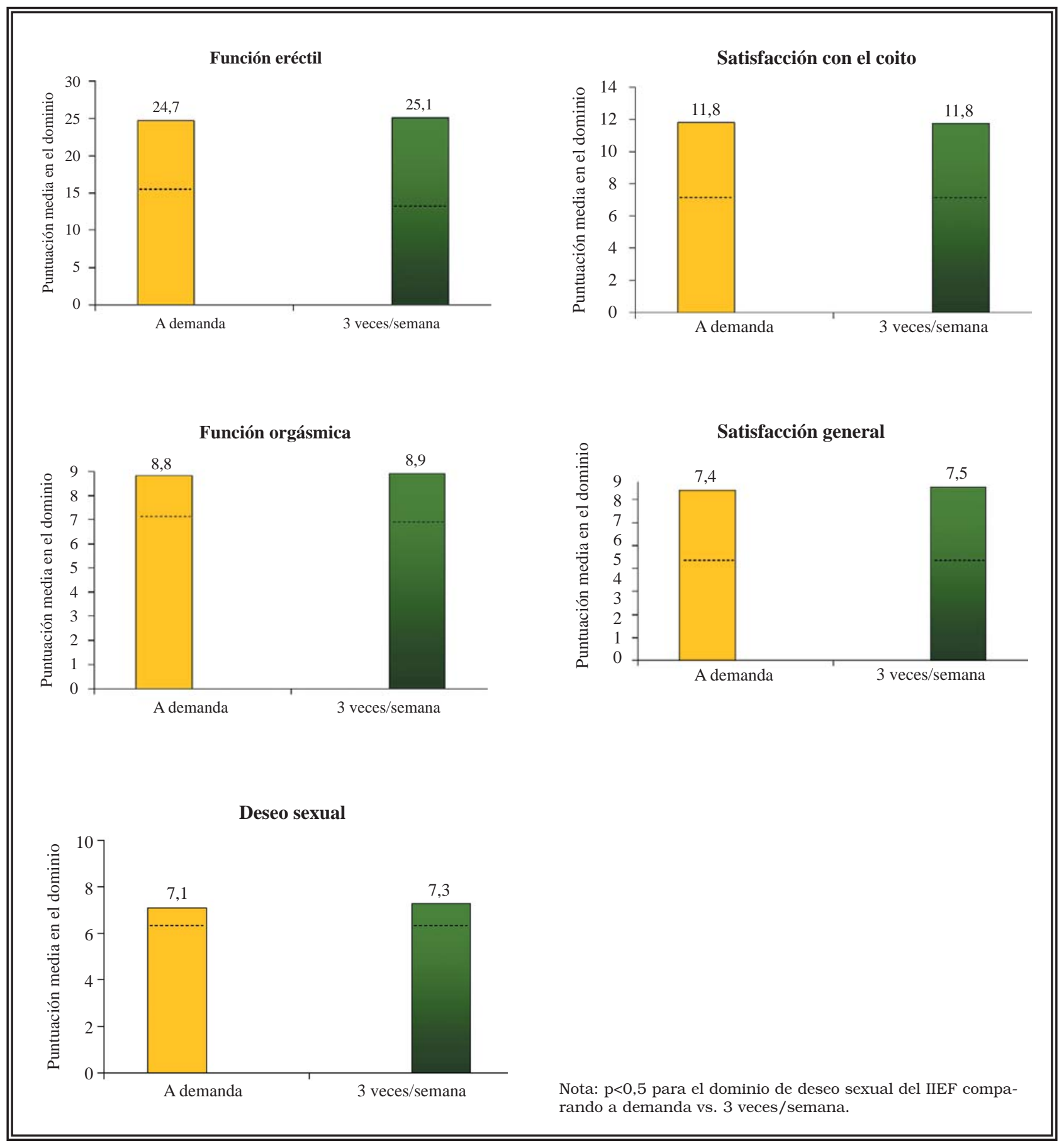

FIGURA 5. Cambio medio respecto al inicio del estudio en los dominios del IEF con ambos regimenes de administración (a demanda y 3 veces/semana).

\section{DISCUSIŌN}

Los inhibidores de la fosfodiesterasa 5 , tradicionalmente administrados "a demanda" en función de las necesidades del paciente, han demostrado una capacidad similar para mejorar la función eréctil. Sin embargo, eficacia y seguridad no siempre van ligados a satisfacción con el tratamiento si no cumplen otras expectativas del paciente tales como la "naturalidad", discreción, y aceptación del tratamiento por parte de la pareja ${ }^{16}$. 


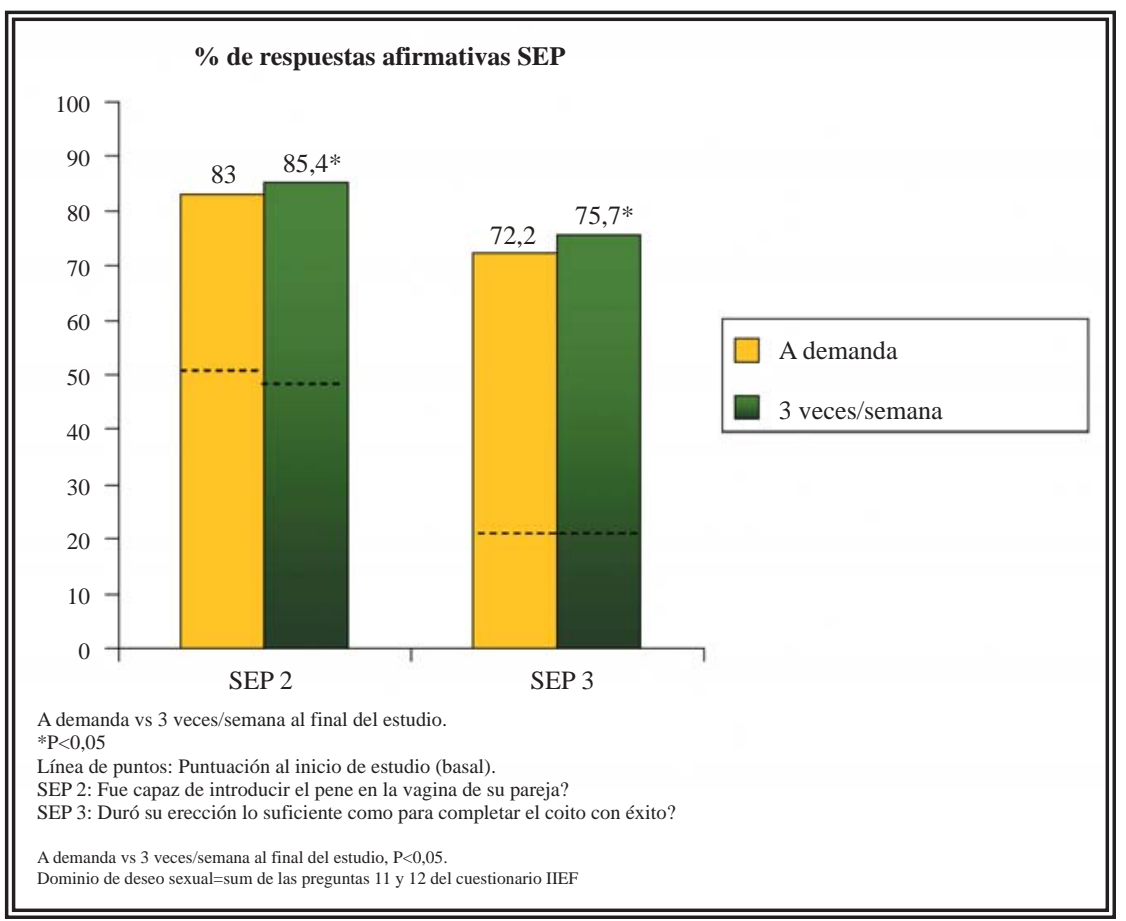

FIGURA 6. Cambio medio respecto al valor basal en el Perfil de Encuentros Sexuales (SEP).

Tadalafilo, un inhibidor selectivo de la PDE5 con una semivida de 17,5 horas, ha demostrado eficacia hasta 36 horas después de la administración (7-14), lo cual permite a los pacientes una amplia ventana temporal para decidir cuándo iniciar la relación sexual con una pareja. Estas propiedades de tadalafilo permitirian a los pacientes optar además por una pauta de dosis fijas como alternativa al tratamiento convencional de la DE a demanda. Una pauta de tratamiento con tadalafilo 3 veces por semana proporciona una cobertura casi continua, que da a los pacientes con DE y a sus parejas una mayor flexibilidad a la hora de decidir cuándo tener relaciones sexuales, y evita la necesidad de coordinar el encuentro sexual con la toma de la medicación. En este estudio aleatorizado, cruzado y abierto con pautas de tratamiento paralelas, se indicó a los pacientes que tomaran tadalafilo según la pauta convencional a demanda y con una nueva pauta de administración 3 veces por semana, con objeto de determinar cuál era la pauta preferida y valorar la eficacia y seguridad del fármaco.

Como muestran los resultados de este estudio, un número mayor de pacientes prefirieron el régimen a demanda $(55,9 \%)$ frente al régimen de 3 veces/semana $(44,1 \%)$, resultados que concuerdan con los hallazgos globales del estudio SURE en el que un $57,8 \%$ de los pacientes prefirieron el régimen a demanda frente al 42,2\% que prefirieron el pautado 3 veces/semana ${ }^{15}$. El número de pacientes que prefirieron este último régimen de tratamiento frente al régimen clásico es bastante conside-

Tabla 2

Relación de acontecimientos adversos aparecidos durante el tratamiento comunicados durante la fase de tratamiento aleatorizado y extensión con una incidencia $\geq 2 \%$.

\begin{tabular}{lccccc}
\hline & \multicolumn{2}{c}{ Fase de tratamiento aleatorizado } & & \multicolumn{2}{c}{ Fase de extensión* } \\
& $\begin{array}{c}\text { Demanda } \\
(\mathrm{N}=418)\end{array}$ & $\mathbf{3}$ veces/sem & Demanda & 3 veces/sem. & No PREF. \\
& $\mathrm{n}(\%)$ & $\mathrm{N}=418)$ & $(\mathrm{N}=205)$ & $\mathrm{n}(\%)$ & $\mathrm{n}(\mathrm{N})=1)$ \\
& $32(7,7)$ & $39(9,3)$ & $6(2,9)$ & $1(0,6)$ & 0 \\
\hline Dispepsia & $17(4,1)$ & $27(6,5)$ & $5(2,4)$ & 0 \\
Cefalea & $15(3,6)$ & $26(6,2)$ & & 0 \\
Dolor de espalda & $7(1,7)$ & $9(2,2)$ & & \\
Dolor en extremidades & $6(1,4)$ & $11(2,6)$ & & \\
Sofocos & & &
\end{tabular}

$P>0,05$ para todos los acontecimientos adversos enumerados empleando la prueba de Fisher's Exact.

*Visita 4: Basal para la fase de extensión. 
Tabla 3

Exposición al fármaco

\begin{tabular}{|c|c|c|c|c|c|c|}
\hline & $\begin{array}{l}\text { A-demanda } \\
\text { (periodo I) }\end{array}$ & $\begin{array}{l}3 \text { veces/ sem } \\
\text { (periodo II) }\end{array}$ & $\begin{array}{l}3 \text { veces/ sem } \\
\text { (periodo I) }\end{array}$ & $\begin{array}{l}\text { A demanda } \\
\text { (periodo II) }\end{array}$ & A-demanda & $\begin{array}{c}\text { Total } \\
3 \text { veces/ semana }\end{array}$ \\
\hline Número de pacientes & 203 & 191 & 213 & 197 & 400 & 404 \\
\hline \multicolumn{7}{|l|}{ Dosis/semana } \\
\hline Media $^{a}$ (DS) & $1, .7(1,0)$ & $2,2(0,7)$ & $2,4(0,9)$ & $1,7(0,9)$ & $1,7(0,9)$ & $2,3(0,8)$ \\
\hline Mediana & 1,5 & 2,5 & 2,8 & 1,7 & 1,6 & 2,6 \\
\hline Rango (min-max) & $0-6,0$ & $0-3,1$ & $0-3,3$ & $0-5,2$ & $0-6,0$ & $0-3,3$ \\
\hline
\end{tabular}

a $P$ valor para la comparación de las medias de las pautas de tratamiento fue $<0,001$.

rable sobre todo teniendo en cuenta que se trata de un esquema de tratamiento nuevo y la tendencia suele ser a favor del clásico. Este resultado puede sugerir que, si se les da la opción de elegir a los pacientes un porcentaje significativo considera deseable y eficaz esta pauta.

Como resultado, ambos regímenes de dosificación, programado y a demanda, condujeron a la mejoría de la función eréctil con respecto al periodo basal, con resultados similares. Sin embargo, el régimen "programado" de 3 veces/semana obtuvo, en general, mejores puntuaciones en los dominios del IIEF, que en el caso del dominio de deseo sexual fue estadísticamente significativo $(\mathrm{p}=0,021)$. De igual manera, fue significativamente mayor el porcentaje de intentos de coito exitosos (SEP3) y el porcentaje de pacientes que normalizaron su función eréctil (IIEF FE $\geq 26$ ) $(\mathrm{P}=0,025)$ Asímismo, los pacientes demostraron mayor satisfacción con la pauta de dosificación de 3 veces/semana según las respuestas a las preguntas $4^{\mathrm{a}}$ y $5^{\mathrm{a}}$ del diario SEP y las puntuaciones del dominio de satisfacción general del IIEF.

Aunque se trata de un estudio abierto, los hallazgos obtenidos son concordantes con los observados en anteriores estudios como se observa en un análisis integrado de estudios aleatorizados, doble-ciego y controlados con placebo realizados con tadalafilo ${ }^{7}$, en donde el 68\% de las tentativas de coito tuvieron éxito en los pacientes que recibieron tadalafilo $20 \mathrm{mg}$ "a demanda" y el $54 \%$ de los pacientes alcanzaron la normalidad en la función eréctil al final del tratamiento. Ambos tratamientos fueron bien tolerados y entre ellos no se observaron diferencias clinicamente significativas respecto a su tolerabilidad.
Estos resultados demuestran que los pacientes y sus parejas pueden realizar el coito en un amplio periodo de tiempo tras la administración de una dosis de tadalafilo administrado independientemente del régimen pautado ("a demanda" / 3 veces por semana). La ansiedad anticipada de fracaso en estos pacientes, adoptando el llamado rol del espectador, puede disminuirse con la pauta programada de Tadalafilo ya que los pacientes se olvidan de un periodo de acción limitado del fármaco. Con un periodo de respuesta tan amplio el facultativo puede recomendar al paciente pautas de dosificación diferentes basándose en las características únicas de tadalafilo.

\section{CONCLUSIÓN}

Un mayor número de pacientes prefirieron Tadalafilo $20 \mathrm{mg}$ en un régimen a demanda hasta un máximo de una dosis/día, que pautado 3 veces/semana, si bien un elevado número de los pacientes (41\%) prefirió esta última opción. Ambos esquemas de dosificación con tadalafilo resultan eficaces y bien tolerados permitiendo al paciente escoger el más adecuado para recuperar la normalidad y espontaneidad en su actividad sexual.

\section{Agradecimientos}

Este estudio clínico ha sido realizado con el patrocinio de Laboratorios Lilly, S.A. Avda. de la Industria, 30. 28108 Alcobendas (Madrid) - España. Los autores desean agradecer la colaboración de los doctores Adrián de la Fuente, Ana Loizaga, Ana Segura Paños, Ander Astobieta, Carlos Pertusa Peña, Emilio Julve, Enrique Cuñat Albert, Esteban Blanco, Fernando Meijide, Francisco Chicote, Francisco Javier Gallo, Francisco Ramada, Jesús García Alonso, Cristóbal López, Jordi Cortada, José Antonio Portillo, José Luis Martín Benito, José Mª Martínez Sagarra, José Manuel 
Barros, José Martínez Jabaloyas, José Ramón Cortiñas, Luis Martínez Piñeiro, Luis Resel Estévez, Luis Rodríguez Vela, Manuel Rivas del Fresno, Manuel Ruiz Ramo, Manuel Varela Salgado, Marceliano García Pérez, Mariano Roselló, Mario Brassesco, Pedro Gutiérrez, Rafael Gutiérrez del Pozo, Rafael Prieto, Ramón Telleria Elorz, Venancio Chantada, y a Elena Bolaños, Paz Sánchez, Elvira Ancillo, María Costi e Inés Lorenzo por su participación en este estudio.

\section{REFERENCIAS}

1. Martin-Morales A, Sanchez-Cruz JJ, Saenz de Tejada I, Rodriguez-Vela L, Jimenez-Cruz JF, Burgos-Rodriguez R. Prevalence and independent risk factors for erectile dysfunction in Spain: results of the "Epidemiologia de la Disfunción Eréctil Masculina” Study J Urol. 2001;166(2): 569-574; discussion 574-575.

2. Guirao Sanchez L, García-Giralda Ruiz L, Sandoval Martínez C, et al. Disfunción eréctil en atención primaria como posible marcador del estado de salud. Factores asociados y respuesta al sildenafilo. Atención Primaria 2002; 30(5):290-296.

3. Wespes E, Amar E, Hatzichristou D, Montorsi F, Pryor J, Vardi Y; European Association of Urology. Guidelines on erectile dysfunction. Eur Urol. 2002;41(1):1-5.

4. Heaton JPW, Hackett G, Savage D, Padley RJ. Patient choice is critical in managing erectile dysfunction. Eur Urol Suppl. 2002;(3):33-37.

5. Chen J, et al. Sildenafil versus the vacuum erection device. Patient preference. J Urol 2000;166:1779-1781.

6. Hackett GI. What do patients expect from erectile dysfunction therapy? European Urology Supplements 2002;1(8):4-11.

7. Carson CC, et al. The efficacy and safety of tadalafil: an update. BJU Int 2004;93(9),1276-1281.

8. Rosen RC, Padma-Nathan H, Shabsigh R, Khalil Saikali, Watkins V, Pullman W. Determining the earliest time within 30 minutes to erectogenic effect after tadalafil 10 and 20 mg: A multicenter, randomized, double-blind, placebo-controlled, at-home study. J Sex Med 2004;1(2):193-200.
9. Brock GB, McMahon CG, Chen KK, Costigan T, Shen W; Watkins $\mathrm{V}$ et al. Efficacy and safety of tadalafil for the treatment of erectile dysfunction: results of integrated analyses. J Urol 2002;168 (4 Pt 1):1332-1336.

10. Padma-Nathan H, Rosen RC, Shabsigh R. Cialis (tadalafil) provides prompt response and extended period of responsiveness for the treatment of men with erectile dysfunction (ED). J Urol 2001 May; 165 suppl: 224.

11. Porst H, Padma-Nathan H, Giuliano F, Anglin G, Varanese L, Rosen R. Efficacy of tadalafil for the treatment of erectile dysfunction at 24 and 36 hours after dosing: a randomized controlled trial. Urology 2003;62(1):121-125; discussion $125-126$

12. Young JM, et al. Tadalafil Improved Erectile Function at Twenty-Four and Thirty-Six Hours After Dosing in Men With Erectile Dysfunction: US Trial. J Androl. 2005;26(3): 310-318.

13. Ficha técnica Tadalafilo.

14. Mirone V, et al. An Evaluation of an Alternative Dosing Regimen with Tadalafil 3 Times/week, for Men with Erectile Dysfunction: SURE Study in 14 European Countries. Eur Urol. 2005;47(6):846-854; discussion 854. Epub 2005 Mar 9.

15. Rosen RC, Riley A, Wagner G, Osterloh IH, Kirkpatrick J, Mishra A. The International Index of Erectile Function (IIEF): a multidimensional scale for the assessment of erectile dysfunction. Urology 1997;49(6):822-830.

16. Hanson-Divers C, Jackon SE, Lue TF et al. Health outcomes variables important in the treatment of erectile dysfunction. J Urol. 1998;159(5):1541-1547.

Dr. A. Cassinello

E-mail: cassinello_alejo@lilly.com

(Trabajo recibido el 3 de mayo de 2006) 